\title{
Stimulus Repetition Effects on Texture-Based Visual Search by Pigeons
}

\author{
Jeffrey S. Katz and Robert G. Cook \\ Tufts University
}

\begin{abstract}
Four experiments investigated the effects of within-session stimulus repetition on texture discrimination. Six pigeons (Columba livia) searched for a contrasting target region (color or shape) randomly embedded within a larger distractor region for food reinforcement. Experiment 1 found that repeating features of the distractors, but not those of the target, across trials increased the accuracy of target localization relative to baseline. Experiment 2 found that subsequently switching the identity of a repeated distractor feature to the target decreased accuracy. Experiment 3 found that the effects of repeating a distractor feature influenced search performance for at least 60 trials after this learning. Experiment 4 found that differential stimulus-outcome relations can produce control by repeated target features. The results are discussed in terms of the factors and strategies involved in the control of avian visual search behavior.
\end{abstract}

Survival is contingent on the accurate search of items in an animal's environment. Birds represent one class of visual animals whose aerial maneuvers and foraging depend on their ability to engage in such search behavior. The visual search of any scene involves both the perception of the current stimuli and a memory for previous stimuli searched for in the same context. The purpose of the following experiments was to explore the interaction of these two fundamental processes in pigeons by examining how the trial-to-trial repetition of features influences the search and localization of targets in an odd-item visual search task.

The visual search task used in the present experiments involved textured visual stimuli (Cook, 1992a, 1992b, 1992c, 1993a, 1993b; Cook, Cavoto, \& Cavoto, 1996; Cook, Cavoto, Katz, \& Cavoto, 1997; Cook, Katz, \& Cavoto, 1998). Visual textures are multidimensional patterns in which global regions are derived from perceptually grouping

Jeffrey S. Katz and Robert G. Cook, Department of Psychology, Tufts University

This research was supported by National Science Foundation Grant IBN-9604359. Preparation of this article was supported by National Institute of Mental Health Postdoctoral Grants NS07373-04 and MH12511-01. This research was part of a doctoral dissertation in psychology submitted to Tufts University by Jeffrey $\mathbf{S}$. Katz. Jeffrey S. Katz thanks his advisor, Robert G. Cook, for his advice support, encouragement, and friendship throughout this project. He also thanks his dissertation committee for their comments on this research: Richard A. Chechille, Raymond S. Nickerson, and Sal A. Soraci, and also thanks Anthony A. Wright for his discussion, guidance, and many useful comments on this article. We also thank Laura Katz for helpful comments on earlier drafts of this article and Brain Cavoto for help in conducting these experiments. Portions of this research were presented at the 66th and 67th meeting of the Eastern Psychological Association.

Correspondence concerning this article should be addressed to Jeffrey S. Katz, who is now at the Department of Neurobiology and Anatomy, University of Texas Medical School at Houston, P.O. Box 20708, Houston, Texas 77225. Electronic mail may be sent to jkatz@nba19.med.uth.tmc.edu. smaller component elements into figure-ground relations (Beck, 1966, 1982; Julesz, 1981; Marr, 1982). In our task, these regions are constructed each trial from randomly selected color and shape elements in which the target region can differ from the distractor region in either color or shape (see Figure 1). The location of the odd target region is randomly positioned on every trial and the pigeons are required to search for and peck at this contrasting target region in order to obtain food reward.

The present experiments explore the strategies or rules used by pigeons when searching for and pecking this odd target region. We think two different processes might be involved. The first process relies on a comparison based on the target's oddity from the distractors. Specifically, this mechanism evaluates the current perception of the display relative to a generalized representation of the typical global relations in these stimuli (e.g., a smaller square odd region embedded within a larger rectangular area; Cook, 1992a, 1993a, 1993b; Cook et al., 1998). This relational rule emphasizes the oddity of the target region relative to the distractor region, which is independent of the absolute identity of the features comprising these regions. It is this relational process that is responsible for the successful transfer of search performance to new examples of textured stimuli (Cook, 1992b).

When the oddity of the target does not readily pop out, however, another type of search process is used. This second process involves a slower search of the display that allows more information about the absolute properties or features of the elements to be encoded. It continues until a pigeon detects a target based on the identity of the elements in the display. This rule is also relational in nature, but depends on the encoding, retention, and search of the specific features encountered within any one display. Because of the itemspecific nature of the latter process, it might be influenced by, for example, the repetition of the display's features across successive trials, especially when perceptual oddity of the display's target is not sufficient to guide search.

This latter item-specific mechanism is highly similar to 


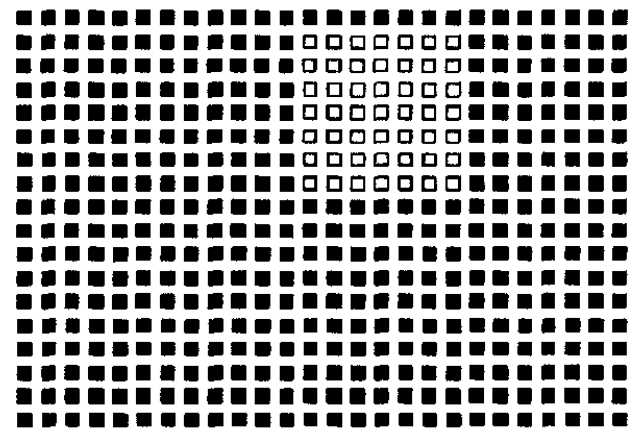

COLOR DISPLAY

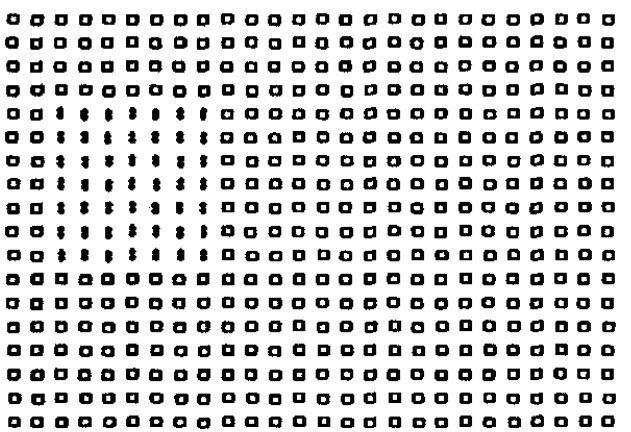

\section{SHAPE DISPLAY}

Figure 1. Examples of color and shape texture displays used in the experiments. The location of the odd target region was randomly selected every trial. In each experiment, the color and shape features used to make up each baseline display were also randomly selected every trial.

those proposed for search image effects. The search image effect occurs when recent encounters with a specific cryptic prey item produce a transient internal change that allows a predator to detect more efficiently subsequent prey items of the same type (Tinbergen, 1960). Such specific search image effects have been frequently studied in the operant chamber using the stimulus repetition procedure. In this type of visual search procedure, the subject searches for the same target item for a number of consecutive trials (the run condition), and performance is then compared to a condition in which different targets are randomly mixed across a series of trials (the non-run condition). This repetition procedure potentially permits a representation of the repeated target to be formed in memory and used to enhance search performance relative to baseline nonrepetition trials, by either decreasing reaction time, (D. S. Blough, 1993; P. M. Blough, 1991, 1996; P. M. Blough \& Lacourse, 1994; Langley, 1996), increasing target detection accuracy (Pietrewicz \& Kamil, 1979; Plaisted, 1997; Reid \& Shettleworth, 1992), or both (P. M. Blough, 1989, 1992; Bond \& Riley, 1991; Vreven \& Blough, 1998).

Whereas our past research has emphasized and examined the role of perceptual grouping and oddity in the successful localization of targets in our texture display search task, in the current experiments, we were more interested in the possible contribution of such item-specific strategies to this type of search task. Using a stimulus repetition paradigm, these experiments investigated the impact of repeating from trial-to-trial the target and distractor components of our textured visual stimuli. Primarily on the basis of the extensive search image literature, we anticipated that repetition of the target regions' features would facilitate its localization. As will be seen, the outcome was quite different and unexpected.

\section{Experiment 1}

Experiment 1 explored the possible contribution of specific search strategies in the processing of textured visual search stimuli using a stimulus repetition paradigm. Each daily session contained a repetition phase that was embedded within a set of baseline trials. The baseline trials consisted of our standard mixture of randomly composed color and shape displays in which the birds had to localize the odd target along either dimension. The repetition phase consisted of repeating a relevant feature from either the target or distractor regions across successive trials. The left half of Table 1 shows an example of target repetition for a run of color displays within a session. In this condition, for example, the color (blue) of the elements forming the target region was repeated, while the color of the elements forming the distractor region and the irrelevant shape element of both regions changed randomly from trial to trial. The right half of Table 1 shows an example of distractor repetition, where the color (red) of the distractor region was repeated, whereas the color of the target region and irrelevant shape of the display's elements changed randomly from trial to trial. These target repetition and distractor repetition conditions were conducted in different sessions. Identical repetition conditions were also tested for shape relevant displays (see Table 2). During target repetition, the shape of the target's elements was repeated across trials, and during distractor repetition the shape of the distractor's elements was repeated across trials. Again, the irrelevant color of these elements varied randomly from trial to trial. The experiment was conducted in two parts. The first part tested the effects of target and distractor repetition on color displays. The second part tested these manipulations on shape displays.

For the target repetition condition, we anticipated performance would improve because the pigeons could learn specifically what to search for across repeated trials (i.e., a search image-like effect). Such a result would indicate that the pigeons sometimes use feature-specific information when searching visual textures. For the distractor repetition condition, we anticipated there might be no change in performance across repeated trials, since the reinforced goal of the task was to localize and peck at the odd target region while ignoring the distractors. 
Table 1

Examples of Target and Distractor Repetition for Color Displays at the Transition From Baseline to the Start of Repetition in Experiment 1

\begin{tabular}{|c|c|c|c|c|c|c|c|c|}
\hline \multirow[b]{3}{*}{ Trial } & \multicolumn{4}{|c|}{ Target repetition } & \multicolumn{4}{|c|}{ Distractor repetition } \\
\hline & \multicolumn{2}{|c|}{ Target } & \multicolumn{2}{|c|}{ Distractor } & \multicolumn{2}{|c|}{ Target } & \multicolumn{2}{|c|}{ Distractor } \\
\hline & Color & Shape & Color & Shape & Color & Shape & Color & Shape \\
\hline \multicolumn{9}{|l|}{ Baseline } \\
\hline 95 & Pink & $\triangle$ & Blue & $\Delta$ & Yellow & a & Cyan & $\mathbf{D}$ \\
\hline 96 & Red & - & Green & - & Brown & $\mathbf{\Delta}$ & Brown & $\Delta$ \\
\hline 97 & Orange & ע & Orange & $r$ & Blue & $\overline{0}$ & Blue & $\rightarrow$ \\
\hline 98 & Cyan & $\checkmark$ & Cyan & $=$ & Pink & $\$$ & Blue & $\$$ \\
\hline 99 & Green & $C$ & Red & $c$ & Green & $\mathbf{T}$ & Green & 80 \\
\hline \multicolumn{9}{|c|}{ Repetition } \\
\hline 100 & Blue & $\mathbf{m}$ & Yellow & $\boldsymbol{n}$ & Orange & + & Red & + \\
\hline 101 & Blue & 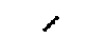 & Brown & , & Pink & $\mathbf{u}$ & Red & $\mathbf{u}$ \\
\hline 102 & Blue & $\$$ & Cyan & $\$$ & Yellow & 1 & Red & 1 \\
\hline 103 & Blue & 3 & Yellow & 3 & Green & 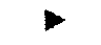 & Red & 1 \\
\hline 104 & Blue & $k$ & Pink & $k$ & White & 0 & Red & 0 \\
\hline
\end{tabular}

\section{Method}

\section{Subjects}

Six White Carneaux pigeons (Columba livia) were tested. No training was necessary, as all birds had extensive experience with searching odd-item textured displays (Cook, 1992a, 1992b, 1992c, 1993a, 1993b; Cook et al., 1996; Cook, Katz, \& Cavoto, 1998). The pigeons were maintained at approximately $80 \%$ of their free-feeding weights. The pigeons had free access to grit and vitamin-enriched water in their individual home cages on a 12 -hr light-dark cycle.

\section{Apparatus}

Testing was done in a painted flat-black Plexiglas chamber (38 $\mathrm{cm}$ wide $\times 36.5 \mathrm{~cm}$ deep $\times 39.5 \mathrm{~cm}$ high). Stimuli were presented on a color computer monitor (NEC, MultiSync 2A; Wooddale, IL) visible through a $25 \mathrm{~cm} \times 17.5 \mathrm{~cm}$ viewing window in the middle of the front panel of the chamber. The bottom edge of the viewing window was $17.5 \mathrm{~cm}$ above the chamber floor. A thin piece of glass mounted in this window protected the monitor. Pecks toward the monitor screen were detected by an infrared touch screen (resolution was $80 \times 48$ locations; EMS Systems, Champaign, IL) mounted behind a $40-\mathrm{mm}$ Plexiglas ledge that went around the inside edge of the viewing window. A 28-V (Model \#1819) houselight was located in the center of the ceiling and was illuminated at all times, unless an incorrect choice was made. A food hopper was located in the middle of the front panel, with its access hole $2 \mathrm{~cm}$ above the chamber floor.

Experimental events were controlled and recorded with a 486-class computer. A video card (VGA Wonder; ATI Technologies, Scarborough, Ontario, Canada) controlled the monitor in the SVGA graphics mode $(800 \times 600$ pixels). Computer-controlled relays (Metrabyte, Taunton, MA) operated the hopper and houselight. Programming was done in QuickBasic (QuickBasic 7.0, 1989) with an attached graphics library (Xgraf, 1989).

Table 2

Examples of Target and Distractor Repetition for Shape Displays at the Transition From Baseline to the Start of Repetition in Experiment 1

\begin{tabular}{|c|c|c|c|c|c|c|c|c|}
\hline \multirow[b]{3}{*}{ Trial } & \multicolumn{4}{|c|}{ Target repetition } & \multicolumn{4}{|c|}{ Distractor repetition } \\
\hline & \multicolumn{2}{|c|}{ Target } & \multicolumn{2}{|c|}{ Distractor } & \multicolumn{2}{|c|}{ Target } & \multicolumn{2}{|c|}{ Distractor } \\
\hline & Color & Shape & Color & Shape & Color & Shape & Color & Shape \\
\hline \multicolumn{9}{|l|}{ Baseline } \\
\hline 95 & Pink & $T$ & Pink & , & Red & $\omega$ & Brown & 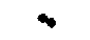 \\
\hline 96 & Orange & $\mathrm{C}$ & Orange & $=$ & Orange & $\Delta$ & White & $\boldsymbol{\Delta}$ \\
\hline 97 & Blue & $\checkmark$ & Cyan & $\checkmark$ & Yellow & $\mathbf{I}$ & Yellow & + \\
\hline 98 & Green & $\Delta$ & Red & $\triangle$ & Green & 3 & Green & 8. \\
\hline 99 & Yellow & $\bar{r}$ & Yellow & $\overline{\mathbf{n}}$ & Blue & 5 & Pink & 5 \\
\hline \multicolumn{9}{|c|}{ Repetition } \\
\hline 100 & White & ᄂ & White & 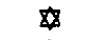 & Violet & 0 & Violet & $\checkmark$ \\
\hline 101 & Red & $\mathbf{L}$ & Red & 0 & Yellow & $\Phi$ & Yellow & $\checkmark$ \\
\hline 102 & Blue & L & Blue & لا & Mauve & $Q$ & Mauve & $\sim$ \\
\hline 103 & Brown & L & Brown & $J$ & Green & 8 & Green & $\checkmark$ \\
\hline 104 & Purple & L & Purple & $c$ & White & 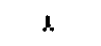 & White & $\checkmark$ \\
\hline
\end{tabular}




\section{Procedure}

Stimulus displays. Each display was $20 \mathrm{~cm}$ wide, $13 \mathrm{~cm}$ high, and contained 468 elements. Individual elements ranged from 3 to $6 \mathrm{~mm}$ in size, based on their respective shape. The elements were placed within a $26 \times 18$ array of spatial locations. Forty-nine of the 468 elements were arranged in a contrasting $7 \times 7$ element target region. For each trial, this target region was randomly placed in one of 240 possible target positions.

Trials were composed from a pool of 680 elements. These elements were formed by pairwise combinations of 20 colors and 34 shapes (this stimulus set can be viewed at http://www.pigeon. psy.tufts.edu/jep/blink/blink_elements.htm; individual shapes are represented in Tables 1, 2,3, and 4). The color and shape trials were generated using the random trial composition procedure (Cook, 1992a). In the 12,920 possible color trials (20 target colors $\times 20$ distractor colors $\times 34$ shapes - 680 uniform combinations) the target elements differed from the distractor elements in their color feature, but not in shape. In the 22,440 possible shape trials, the target elements differed from the distractor elements in their shape feature, but not in color. Before the experiment, this pool of 35,360 displays was regularly being tested in daily 180-trial sessions, composed of 90 randomly chosen color and 90 randomly chosen shape displays.

Discrimination testing procedure. Each discrimination trial began with a peck to a white circular ready signal $(2 \mathrm{~cm}$ in diameter) randomly placed within the upper two thirds of the display area. This response darkened the computer screen for $.1 \mathrm{~s}$, followed by presentation of a texture display. A trial was considered correct if 5 pecks were directed to the target region before 5 pecks were directed to the distractor region. This "voting" method results in a minimum of 5 pecks and a maximum of 9 pecks to each display. Pecks directed to the target region and the distractor elements bordering the target region were treated as target-directed responses. On completion of the peck requirement, the texture display was turned off. Correct target localization responses were rewarded with 2 -s access to mixed grain. Incorrect responses were punished with a 10-s timeout by extinguishing the houselight. An illuminated 5-s intertrial interval (ITI) followed reward or punishment.

Design. The experiment consisted of 32 sessions. Each session contained 180 trials. The first 20 trials of every session were warm-up trials in which the pigeons were required to peck the target five times regardless of the number of pecks to the distractor region. These warm-up trials always resulted in reward and were excluded from all data analyses. From Trials 21 to 99 and from Trials 161 to 180 , birds were tested with randomly intermixed color and shape baseline displays.

Repetition conditions for color displays. Target and distractor repetition occurred from Trials $100-160$. Table 1 shows example trials of the two test conditions for the five trials before and after the start of repetition. During target repetition, the color feature of the target elements was repeated across trials while the color feature of the distractor elements changed at random. During distractor repetition, the color feature of the distractor elements repeated across trials while the color feature of the target elements changed at random. In both conditions, the irrelevant shape feature randomly changed across trials. Repetitions of the color features were tested for 16 sessions, with distractor and target conditions tested in different sessions in randomized two session blocks.

Repetition conditions for shape displays. These test conditions were identical to those for color displays, except a series of shape trials was presented from Trials $100-160$. Table 2 shows trials of the two test conditions for the five trials before and after the start of repetition. During target repetition, the shape feature of the target elements was repeated across trials while the shape feature of the distractor elements changed at random. During distractor repetition, the shape feature of the distractor elements was repeated across trials while the shape feature of the target elements changed at random. In both conditions, the irrelevant color feature randomly changed across trials. Repetitions of the shape features were tested for the last 16 sessions, with distractor and target conditions tested in different sessions in randomized two session blocks.

\section{Results}

Figure 2 presents mean target localization accuracy for the repetition conditions of color (upper panel) and shape (lower panel) displays across trials for distractor (circle symbols) and target (triangle symbols) repetition sessions. The open symbols denote mean baseline performance for color displays in the top panel (shape trials excluded) and for shape displays in the bottom panel (color trials excluded). Filled symbols denote performance during the repetition phase. In all experimental analyses, trials in which first-peck reaction times exceeded $8 \mathrm{~s}$ were eliminated (less than $1 \%$ of the trials).

Separate two-way repeated measures analyses of variance (ANOVAs) - Repetition Condition (distractor vs, target) $\times$ Trial Block $(21-99,100-115,116-130,131-145,146-$ 160)-were conducted to evaluate the effects of shape and color repetition. For shape repetition the interaction between repetition condition and trial block was significant, $F(4$, $20)=2.99, p<.05$. This interaction was due to the increase in target localization accuracy from baseline (Trials 21-99) for distractor repetition but not for target repetition. Separate trend analyses of trial-block for shape distractor and target repetition confirmed the interaction, yielding a significant positive linear trend only for distractor repetition, $F(1,5)=$ $17.72, p<.01$. For color repetition, the interaction between repetition condition and trial block was not significant, $F(4$, $20)=.86, p>.05$. A main effect of repetition condition, $F(1,5)=6.7, p<.05$, was found, however, with color distractor repetition $(80.5 \%)$ resulting in higher accuracy than target repetition $(77.7 \%)$.

To judge the effects of repetition on baseline performance, we analyzed data from both before and after repetition with a three-way repeated measures ANOVA: Dimension (color vs. shape) $\times$ Repetition Condition (Distractor vs. Target) $\times$ Trial Block (21-99, 161-180). A main effect of dimension, $F(1,5)=60.5, p<.001$, was found due to the generally higher accuracy with color displays $(78.9 \%)$ than shape displays $(67 \%)$. There were no main effects or other interactions among the other factors, indicating that repetition per se had no generalized effect on target localization performance.

The next analysis examined the role of dimensional repetition per se in the above effects. That is, how did repeating the relevant dimensional cue (color or shape) itself, which did covary with the repetition of the features, improve search performance? The failure to see any increase in performance with target repetition suggests that repeating dimensional information per se does not influence search to any great extent. After the completion of Experiment 4, however, we did collect some observations in which the 


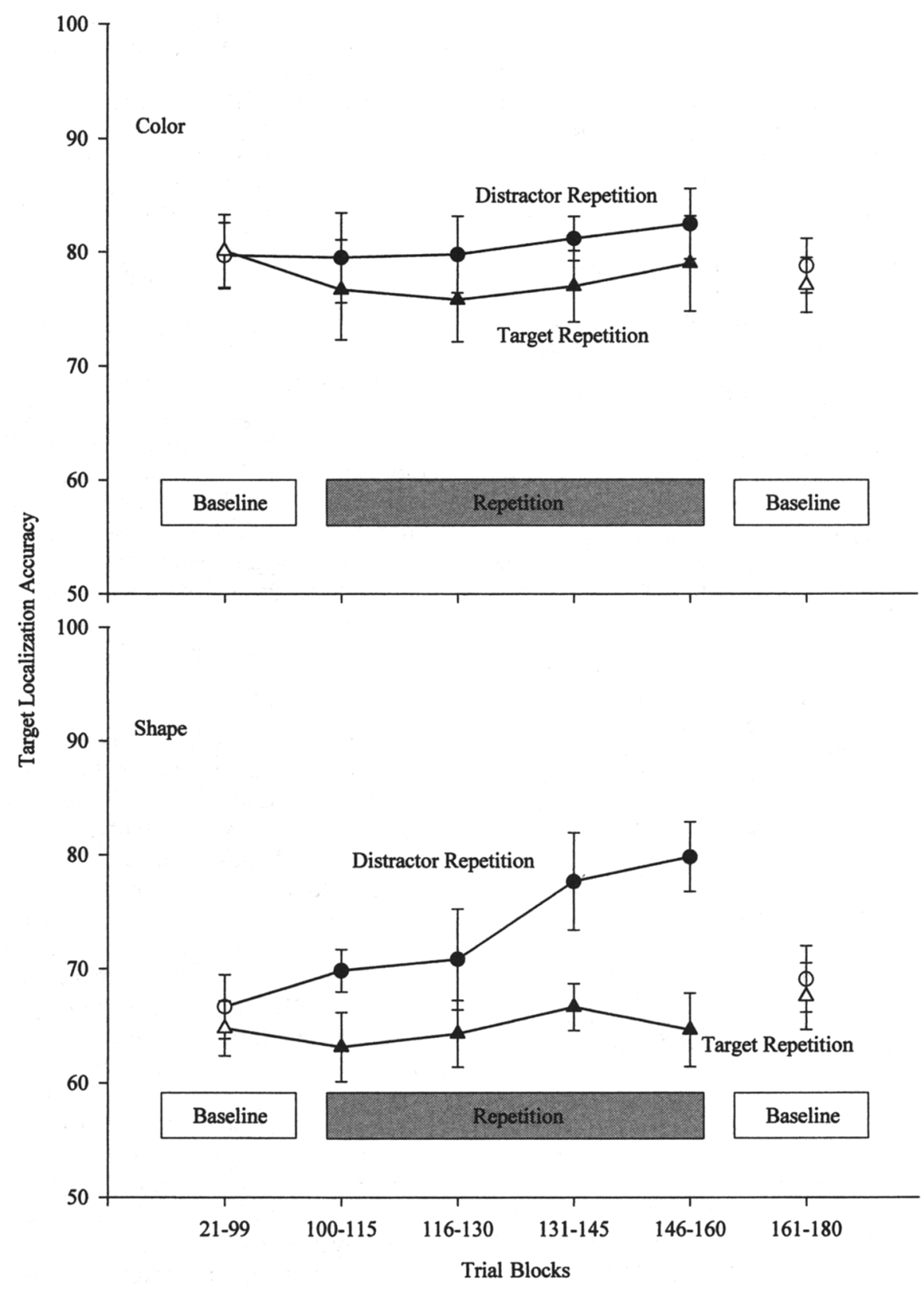

Figure 2. Mean target localization accuracy and standard errors of the mean across successive trial blocks within a session for Experiment 1. The repetition conditions for color and shape displays are respectively shown in the top and bottom panels. For baseline (open symbols), only color (top panel) and shape (bottom panel) performance is represented.

repetition phase consisted of only runs of either shape or color displays with no specific feature repetition included. Across 12 sessions of testing, there was no significant change in performance across trial block (11-99, 100-115, $116-130,131-145,146-160$ ) for shape display repetition $(71.5 \%, 68 \%, 70.7 \%, 71.5 \%, 69 \%$, respectively) or color display repetition $(81.2 \%, 82 \%, 83.3 \%, 85.5 \%, 80.8 \%$ respectively), as determined by one-way repeated measures ANOVAs, both $F \mathrm{~s}(4,20)<1.3, p \mathrm{~s}>.05$.

\section{Discussion}

Experiment 1 revealed that repetition of the relevant features of the distractor region resulted in a monotonic increase in target localization accuracy, whereas repetition of target features showed no comparable effect. This distractor repetition effect occurred only with tests of shape stimuli. These results reveal a new factor in avian visual search involving memory for distractor identity. It further indicates 
that feature-specific search strategies are also involved in our textured visual search task.

The difference in the effects of repetition for each of the dimensions most likely reflects the pigeons' generally superior ability to discriminate color information. In the previous search image experiments, it has commonly been found that cryptic stimuli are more susceptible to the effect of repetition than are conspicuous stimuli (Bond, 1983; Bond \& Riley, 1991; Langley, 1996; Langley, Riley, Bond, \& Goel, 1996; Reid \& Shettleworth, 1992). We hypothesize that much the same thing is true in the present context due to two different routes used by the birds in locating the target (for similar arguments see Bond, 1983; Reid \& Shettleworth, 1992). When the grouping of similar stimuli produces globally distinct regions, the pigeons perform a rapid and accurate relational discrimination based on the global oddity of the target region. Little information is retained in memory regarding the specific features of such displays, and consequently there is little benefit from repeating these values across successive trials. On the other hand, when perceptual grouping does not result in distinctive regions, a more attentive, controlled item-specific search of the display is needed. Because of this more focused use of resources, it allows experienced features to remain active or primed in memory for a longer period of time, and it results in the accuracy benefits across repetition observed above. Because of the generally greater discriminability of the color displays, the need to engage in the more elaborated form of feature search was called on less often.

Much of the above is not at odds with what has been written about the benefits of repetition concerning the phenomenon of a specific search image. What is different about the present search effect is that it was exclusively associated with the features of the distractor region and not of the target. This is different from what has been found before, and it suggests that the specific search strategy used by the birds in the present case is governed more by a "distractor avoidance" rule rather than a "target approach" rule.

Not only is this different from previous search image findings, but is it also counterintuitive from a conditioning perspective based on temporal contiguity and duration. From this viewpoint, one might have expected target repetition to produce an increase in accuracy relative to distractor repetition. First, the target features are always the last items pecked just prior to reward, thus the contiguity between pecking or observing the target features and reward is much closer in time than for the distractor features. Second, the multiple peck requirement to the target ensures that on trials leading to reward, more time is spent in the presence of the target features than in avoiding the distractors. It took about $2226 \mathrm{~ms}$ on average to complete the peck requirement to the target after locating it, whereas it took about $1338 \mathrm{~ms}$ to first locate it, as measured by the reaction time to the first correct peck. On the basis of the longer duration of interacting with its features and its closer temporal proximity to reward, it seems that the trial-to-trial repetition of target features should have facilitated a stronger generalized approach responding, similar in nature to autoshaping (Brown \&
Jenkins, 1968) and the feature-positive effect (Jenkins \& Sainsbury, 1970). Clearly, this outcome did not occur, as performance was controlled by the more temporally remote features of the distractor elements.

Why did the features of the distractor region play such a role in the present search task? One likely reason is the distractor region's much larger size relative to the target region. When a display is presented, the pigeons almost surely encounter distractor elements when first seeing the display. Further, in the standard baseline condition that these birds had extensively experienced, the identity of the target was always unknown from trial to trial. As such, successfully learning to avoid distractors may be more crucial to such a task then ones in which the target item(s) are predictable. Thus, when an odd target is not readily perceived by the pigeons it may be more profitable to encode the identity of the first or most frequently encountered item and then search until a different looking set of elements is encountered. When these contrasting nondistractor items are finally encountered, the pigeons might only then need to respond "automatically" to them without further processing or remembering the specific identity or features of this target region, and perhaps causing our failure to find target repetition effects in the current experiment.

\section{Experiment 2}

Experiment 2 tested whether the pigeons were specifically learning to avoid repeated distractors. If the pigeons were learning to avoid the repeated distractor feature, then if this repeated feature were to have its role suddenly reversed, now appearing in the target, it should result in a brief decline in target localization accuracy, as it is now inappropriately not pecked at. This idea was tested in Experiment 2 by using a discrimination reversal procedure. In this procedure, after a distractor feature had been repeated, it was immediately switched to being a repeated target feature within the same session. In comparison to a shift to a baseline condition, we were interested in how the pigeons reacted to these rolereversed shifted features, following both target and distractor repetition.

\section{Method}

\section{Animals and Apparatus}

The same pigeons and apparatus were used as in Experiment 1.

\section{Procedure}

In this experiment, repetition conditions were conducted using only shape trials. Repetition conditions (Trials 71-170) were divided into two contiguous stages lasting 60 (Stage 1) and 40 (Stage 2) trials. Stage 1 consisted of distractor or target repetition conditions conducted just as before. Stage 2 consisted of either discrimination reversal or shape baseline trials. Discrimination reversal following distractor repetition consisted of repeating the same feature from Stage 1, but now tested and repeated as part of the target region's elements. Discrimination reversal following target repetition consisted of repeating the same feature from Stage 1 , but now tested and repeated as part of the distractor region's 
elements. The shape baseline condition followed target or distractor repetition with a Stage 2 that consisted only of randomly generated shape displays. In every case, the irrelevant color of the elements was varied at random from trial to trial. All together there were four repetition conditions: distractor repetition trainingtarget testing (Stage 1-Stage 2), distractor repetition trainingshape baseline, target repetition training-distractor testing, and target repetition training-shape baseline. Table 3 provides example trials of the transition from Stage 1 to Stage 2 for these four conditions. Thirty-two sessions were conducted, containing eight randomized blocks of the four conditions. All other session and stimulus details were identical to Experiment 1, except that sessions were now 190 trials in duration, and three new shape features had been added between Experiment 1 and 2 .

\section{Results and Discussion}

The left side of the top and bottom panels of Figure 3 present mean target localization accuracy for distractor and target repetition training, respectively. These data are similarly arranged to the previous experiment. The open symbols denote mean baseline performance for shape displays only. Filled symbols denote shape repetition trials. The two bars in the top panel of Figure 3 show mean target localization accuracy for the first 20 trials of shape baseline (white) and the feature reversed target testing (black) following distractor training, while the bottom panel shows the same data for shape baseline (white) and the feature reversed distractor testing (black) following target training.

The pigeons significantly avoided to a greater degree a target region containing a formerly repeated distractor feature than with a comparable set of baseline shape trials. Following distractor repetition training, there was a significant difference between accuracy in the target testing and shape baseline conditions, $F(1,5)=22.7, p<.005$ (one-way repeated measures ANOVAs used throughout this experiment). A similar analysis comparing performance after target repetition training was not significant, $F(1,5)=$ $1.9, p>.05$. This reversal effect for distractor training was short-lived, with the pigeons returning to baseline levels of accuracy within the next 20 trials. Accuracy on the last 20 trials (Trials 151-170) for shape baseline (72.3\%) and target testing $(72 \%)$ was not significantly different. Following target training, performance on the last 20 trials (Trials 151-170) for shape baseline (70.3\%) and distractor testing (73.2\%) was not significantly different.

Separate ANOVAs on trial block (21-70, 71-85, 86-100, 101-115, 116-130) were conducted for distractor and target repetition training. Both the main effect for distractor, $F(4,20)=7.8, p<.001$, and target repetition training, $F(4$, $20)=3.2, p<.05$, were significant. Albeit small relative to distractor repetition, why was there an effect of target repetition in this experiment but none in Experiment 1? It may be that target feature processing was enhanced due to the very use of the discrimination reversal procedure. Perhaps because the specific identity of the target's features were made more relevant in one sense by the role reversal shift following distractor repetition training, the pigeons began to stop automatically responding to the target region and started to pay more attention to its properties.
In summary, this experiment revealed that once the pigeons learned to avoid a repeated distractor feature, they then avoided this same feature when it subsequently appeared in the target. This fact confirms the pigeons were using a feature-specific avoidance strategy and that this information originated from their experience in processing the distractor regions of the displays.

\section{Experiment 3}

The results of Experiment 2 suggest that the effect of repetition has a somewhat long lasting, but not permanent, influence on search. For a trial-to-trial repetition effect to occur, the memory for a previous trial must last at least one trial. The memory for target-mediated search images has generally been found to be short lived, dissipating after $30 \mathrm{~s}$ (Langley et al., 1996). In Experiment 3, we addressed the question of how long the pigeons remained under stimulus control from the repeated distractor feature. This was tested by presenting the feature of a previously repeated distractor on probe trials at various points later in a session. Accuracy on these subsequent probe trials assessed how long the pigeons were still able to access and utilize their memory of the previously repeated feature.

\section{Method}

\section{Animals and Apparatus}

The same pigeons and apparatus were used as in Experiment 2.

\section{Procedure}

Again, repetition conditions were conducted using only shape trials. For the first 28 sessions of testing, these 60 shape repetition trials occurred from Trials 51 to 110 and for the last 28 sessions from Trials 41 to 100 of each session. Memory probe tests then occurred at various points after this distractor repetition phase. These tests used the same shape feature to form their distractor region as presented earlier during the repetition phase. Again, the repeated feature was randomly selected at the beginning of each session from the entire set of shapes. Thus, when selected, this feature occurred only in the distractor repetition condition and probe tests of that session. Four different probe test conditions were conducted. These tests occurred 5 (P5), 10 (P10), 30 (P30), or 60 (P60) trials after the completion of the distractor repetition phase. Probe tests occurred for P5 on Trial 115, for P10 on Trial 120, for P30 on Trials 129 and 130, and for P60 on Trials 159 and 160. Additional probe tests were sometimes presented after these tests in some of the conditions, but these contaminated observations are not included in the analyses below. Only the first probe test of any condition was used in assessing memory for the repeated distractor feature.

The experiment consisted of 56 sessions. P5 and P10 testing was conducted during the first 28 sessions in randomized blocks of two. $\mathrm{P} 30$ and $\mathrm{P} 60$ testing was conducted during the last 28 sessions in randomized blocks of two. All other aspects of the experiment were identical to the previous experiments, except that four novel shape features had been added between the end of Experiment 2 and the start of Experiment 3, increasing the total number of displays to $48,380(15,580$ color displays and 32,800 shape displays), and 12 


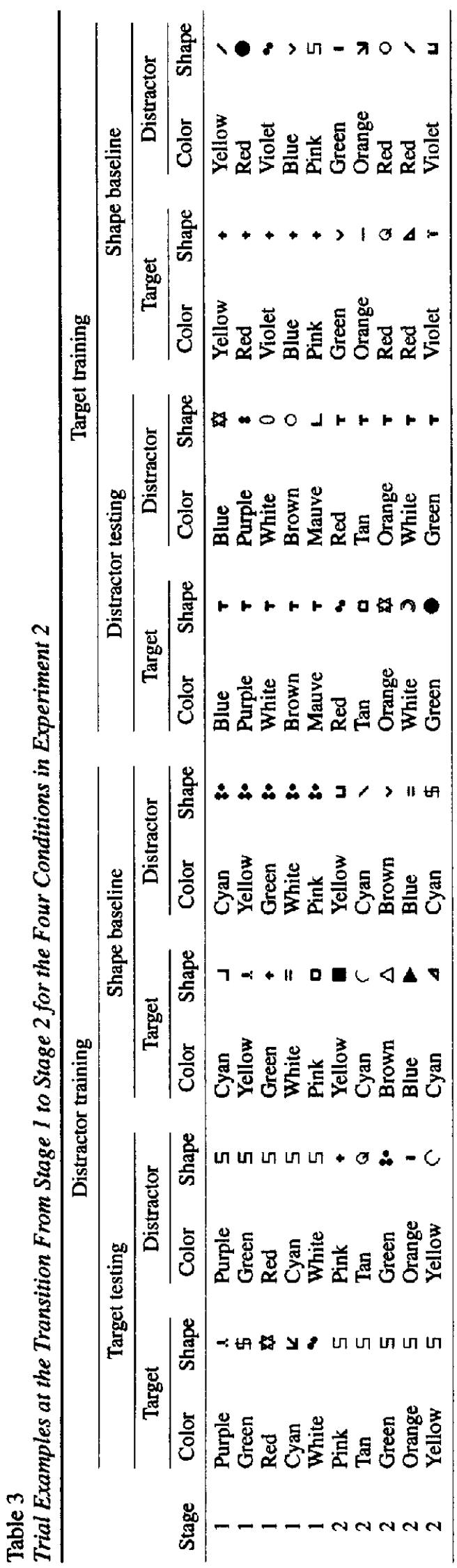

line orientation displays, conducted for other reasons, were tested at the end of each of these 180-trial sessions.

\section{Results and Discussion}

The left side of Figure 4 shows the now expected distractor repetition effect. Open symbols denote shape baseline trials and filled symbols denote distractor repetjtion. For P5 and P10 sessions (triangles), trials were grouped into the following successive trial blocks: $21-50,51-65$, $66-80,81-95,96-110$. For P30 and P60 sessions (circles), trials were grouped into the following successive trial blocks: $21-40,41-55,56-70,71-85,86-100$. Again, the birds showed a significant increase in target localization accuracy with distractor repetition.

The right side of Figure 4 shows probe test performance (filled symbols) in comparison to shape baseline. The shape baseline data points in the right side of Figure 4 show mean performance for the four shape trials most closely preceding and following the probe tests during their respective sessions (performance on color displays were excluded), and were selected to assess memory probe test performance. For P30 and P60 sessions, only the first probe test trial from each testing session is plotted, although mean performance on the second probe test trial for $\mathrm{P} 30(79.5 \%)$ and $\mathrm{P} 60(81.8 \%)$ did not significantly differ from the first test trial, $F_{\mathrm{s}}(1,5)<1.5$, $p$ s $>.05$.

Mean accuracy on probe tests (78.5\%) was higher than on the post-repetition shape baseline trials $(72.8 \%)$. A two-way repeated measures ANOVA for probe test (P5, P10, P30, P60) and trial type (probe, shape baseline) confirmed this, yielding only a significant main effect of trial type, $F(1,5)=$ $8.1, p<.05$. This indicates the pigeons were remembering the previously repeated distractor feature into the postrepetition period. The memory for the repeated feature did decline initially, as evidenced by significantly lower performance on the probe tests in comparison with the last 15 trials of distractor repetition, $84 \% ; F(1,5)=31.7, p<.005$. But the lack of any interaction or main effect of probe test, however, suggests that the memory for the repeated distractor feature is relatively stable after that, influencing performance for up to 60 trials after its last appearance. Taking into account stimulus duration, reward, timeouts, ITI, and the pigeons' time to peck the warning signal for these 60 trials, an estimate for the elapsed time between these intervals would be about $30 \mathrm{~min}$.

Considering the number of intervening, and potentially interfering, trials and the elapsed time between the end of repetition and the probe tests, it suggests that the pigeons' memory for the repeated distractor feature was robust. Earlier work exploring the effects of duration on memory of a repeated target has found memory for target features to dissipate between $30 \mathrm{~s}$ and $3 \mathrm{~min}$. (Langley et al., 1996). Consistent with this temporal window was a study in which sequentially primed targets were remembered for all durations tested from $2.5 \mathrm{~s}$ to $17.5 \mathrm{~s}$ (P. M. Blough, 1991). Although in the present experiment memory for repeated distractor features seemed to be much longer than that found for target features in these earlier studies, it is difficult to 


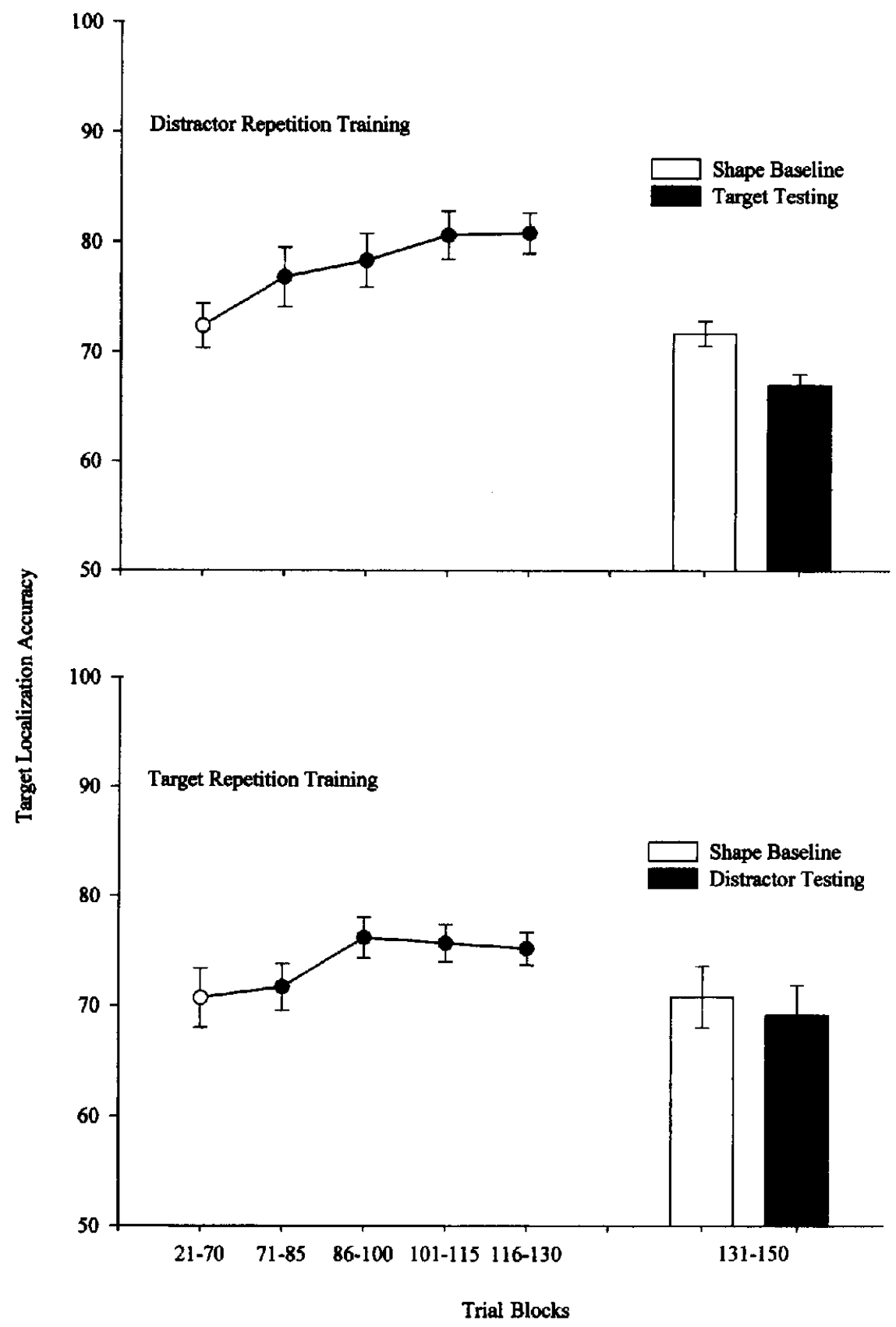

Figure 3. The left side of the top and bottom panels, respectively, present mean target localization accuracy and standard enrors of the mean for distractor and target repetition training across successive trial blocks within a session for Experiment 2. The bar graph in the top panel presents mean target localization accuracy and standard errors of the mean following distractor repetition for the first 20 trials of target testing and shape baseline. The bar graph in the bottom panel presents mean target localization accuracy and standard errors of the mean following target repetition for the first 20 trials of distractor testing and shape baseline. 


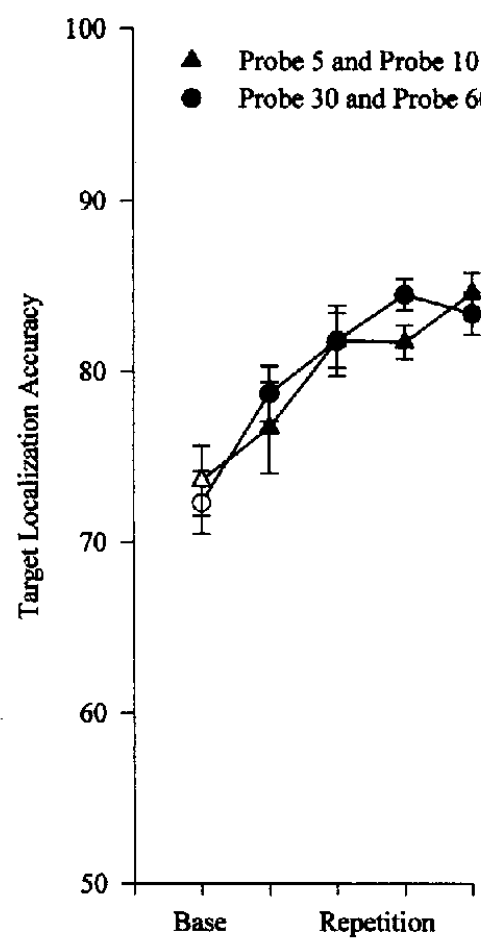

Trial Blocks
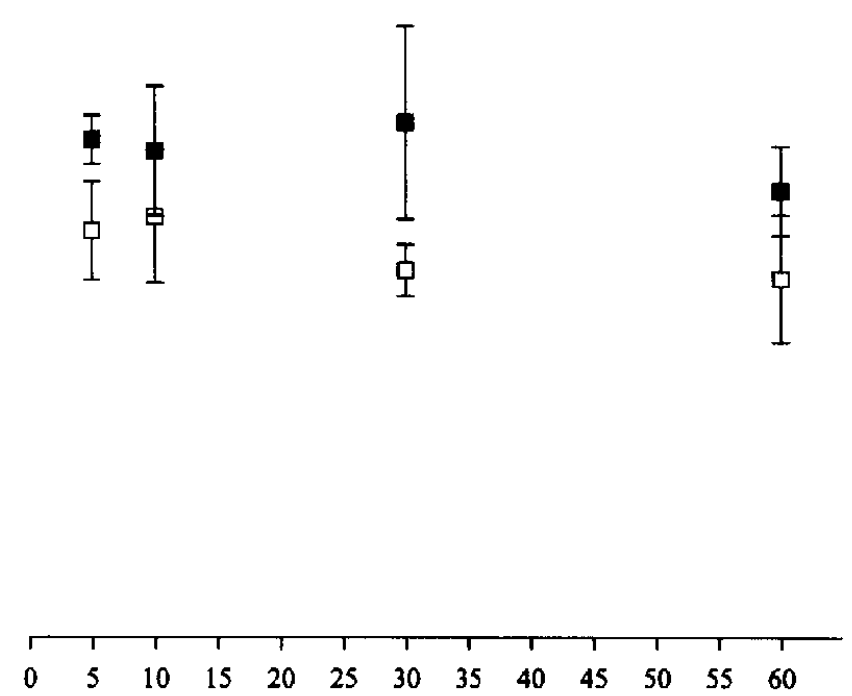

Post-Repetition Trial

Figure 4. The left side represents mean target localization accuracy and standard errors of the mean across successive trial blocks within a session for shape baseline (open symbols) and distractor repetition (filled symbols) of Experiment 3. The right side represents mean target localization accuracy and standard errors of the mean for probe tests (filled symbols) and shape baseline (open symbols). After the end of distractor repetition, the abscissa represents the trial number within a session on which memory probe tests occurred. For this post-repetition period, shape baseline represents tests of shape displays that occurred within the four trials preceding and following the probe tests from their respective sessions.

draw any strong conclusions about this difference because of numerous procedural differences between the studies (e.g., long vs. short runs of repetition, the presence or absence of intervening trials, odd-item vs. fixed-item searches).

\section{Experiment 4}

Experiments 1 and 2 showed that there was less stimulus control elicited from repeated target than distractor features. On the basis of previous research suggesting the efficacy of target repetition, this was a somewhat surprising result. Experiment 4 attempted to help clarify the reasons for this discrepancy. In it, we attempted to enhance attention to the target by differential reinforcing some of the target features. This was implemented using a two-feature repetition procedure. In one condition, the "differential target condition," two different target features were repeated within the same session in which only one of the target features was reinforced. The top half of Table 4 shows an example of six consecutive trials from this condition, where the target feature $\mathbf{Q}$ is reinforced as usual and the target feature $\$$ is not. Displays containing the reinforced target were rewarded for accurate target localization (i.e., pecks to the target region) and punished for incorrect responses (i.e., pecks to the distractor region). Displays containing the nonreinforced target feature, both correct and incorrect target localization responses resulted in the end of a trial (i.e., no food delivery or timeout). In a similar condition, the "differential distractor condition," two different distractor features were repeated within the same session in which only one of the distractor features was reinforced. The bottom half of Table 4 shows examples of six consecutive trials from this condition, where the distractor feature $=$ is reinforced as usual (i.e., rewarding correct target localization and punishing incorrect target localization) and the distractor feature is not rewarded. In another condition, the "nondifferential target condition," two different target features were repeated within the same session in which both target features were reinforced in the same manner as in Experiments 1 and 2. In another condition, the "nondifferential distractor condition," two different distractor features were repeated within the same session in which both distractor features were reinforced in the same manner as in Experiments 1,2 , and 3 .

If the pigeons can remember two features simultaneously 
Table 4

Examples of a Portion of Trials During the Target and Distractor Differential Reinforcement Conditions From Experiment 4

\begin{tabular}{|c|c|c|c|c|c|}
\hline \multirow[b]{2}{*}{ Condition and trial } & \multirow[b]{2}{*}{ Reinforced $^{a}$} & \multicolumn{2}{|c|}{ Target } & \multicolumn{2}{|c|}{ Distractor } \\
\hline & & Color & Shape & Color & Shape \\
\hline \multicolumn{6}{|l|}{ Target } \\
\hline 31 & Yes & Blue & 口 & Blue & $\Delta$ \\
\hline 32 & No & Red & 事 & Red & 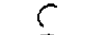 \\
\hline 33 & Yes & Orange & a & Orange & 0 \\
\hline 34 & No & Green & 车 & Green & $y$ \\
\hline 35 & No & Purple & \$ & Purple & + \\
\hline 36 & Yes & Yellow & 口 & Yellow & $\checkmark$ \\
\hline \multicolumn{6}{|l|}{ Distractor } \\
\hline 31 & No & Cyan & 1 & Cyan & $\$$ \\
\hline 32 & Yes & Pink & 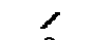 & Pink & $=$ \\
\hline 33 & Yes & Violet & 0 & Violet & $=$ \\
\hline 34 & No & Brown & $\mathbf{u}$ & Brown & $\$$ \\
\hline 35 & No & Blue & $\mathbf{T}$ & Blue & $\$$ \\
\hline 36 & Yes & Orange & 0 & Orange & $=$ \\
\hline
\end{tabular}

a"Yes" indicates that a target choice was rewarded and a distractor choice was punished. "No" indicates that both target and distractor choices were not reinforced.

within the same session, then the nondifferential reinforcement conditions should produce the same results as before That is, the pigeons should learn to avoid the two repeated distractor features within a session, but not search for the two repeated target features. For the differential reinforcement conditions, because the different features now predicted whether the pigeons could receive food or not on a trial, the processing of the identity of these features would become more significant for the birds. In the case of the differential target condition, we thought it would directly promote the processing of the target's features. If so, we should then see effects of target repetition on these features. For the differential distractor condition, because the pigeons already were processing the distractors of the displays, this increased significance should not change their search behavior.

\section{Method}

\section{Animals, Apparatus, and Stimuli}

The same 6 pigeons, apparatus, and stimuli used in the previous experiments were used in this experiment.

\section{Procedure}

Target and distractor repetition were similar to the previous experiments, but now two different shape features were randomly selected each session and repeated in randomized blocks of two. Each repeated feature occurred 60 times. These 120 target or distractor repetition trials occurred from Trials 31 to 150 of each session. Again, repeated features were only presented during repetition trials within a particular session. During the two nondifferential reinforcement conditions, both repeated features were reinforced exactly like in Experiment 1. During differential reinforcement conditions, only trials containing one of the two repeated shape features was rewarded. Accurate localization of the target region on these trials ended in 2-s food reward and incorrect localization produced a 10-s timeout. Trials containing the other repeated shape feature resulted in extinction (i.e., no food reward and no timeouts) regardless of the accuracy of the target localization. The completion of the peck requirement on these nonreinforced trials simply ended the trials and started the ITI for the next trial.

The experiment lasted 84 sessions. All together, four conditions were tested using the two-feature repetition procedure: differential distractor repetition, nondifferential distractor repetition, differential target repetition, and nondifferential target repetition. The four different conditions occurred in the following testing block order with their respective number of sessions in parentheses: nondifferential distractor (18), differential distractor (6), differential target (6), nondifferential target (18), differential target (6), nondifferential target (6), differential target (6), nondifferential target (6), differential distractor (6), and nondifferential distractor (6). Each session contained 188 trials. The first 10 trials of each session were warm-up trials. The last 18 trials from each session consisted of displays constructed from different line orientations. As these displays were not germane to the current experiment, they have been eliminated from the analyses. All other details were identical to the previous experiments.

\section{Results}

The separate blocks of differential and nondifferential testing on two-feature distractor and target repetitions are shown in Figures 5 and 6, respectively. Consider first the pattern of effects in the differential blocks. For both distractor and target repetition, there was a divergence in accuracy between the reinforced, $R(+)$, and nonreinforced features, $N R(-)$, in the differential blocks. For distractor repetition, there was an increase in target localization accuracy from baseline (Trials 11-30) for the nonreinforced distractor feature, whereas the reinforced distractor feature showed little or no increase across trials. For target repetition, there was a decrease in accuracy from baseline for the nonreinforced target feature, whereas there was a clear increase in 


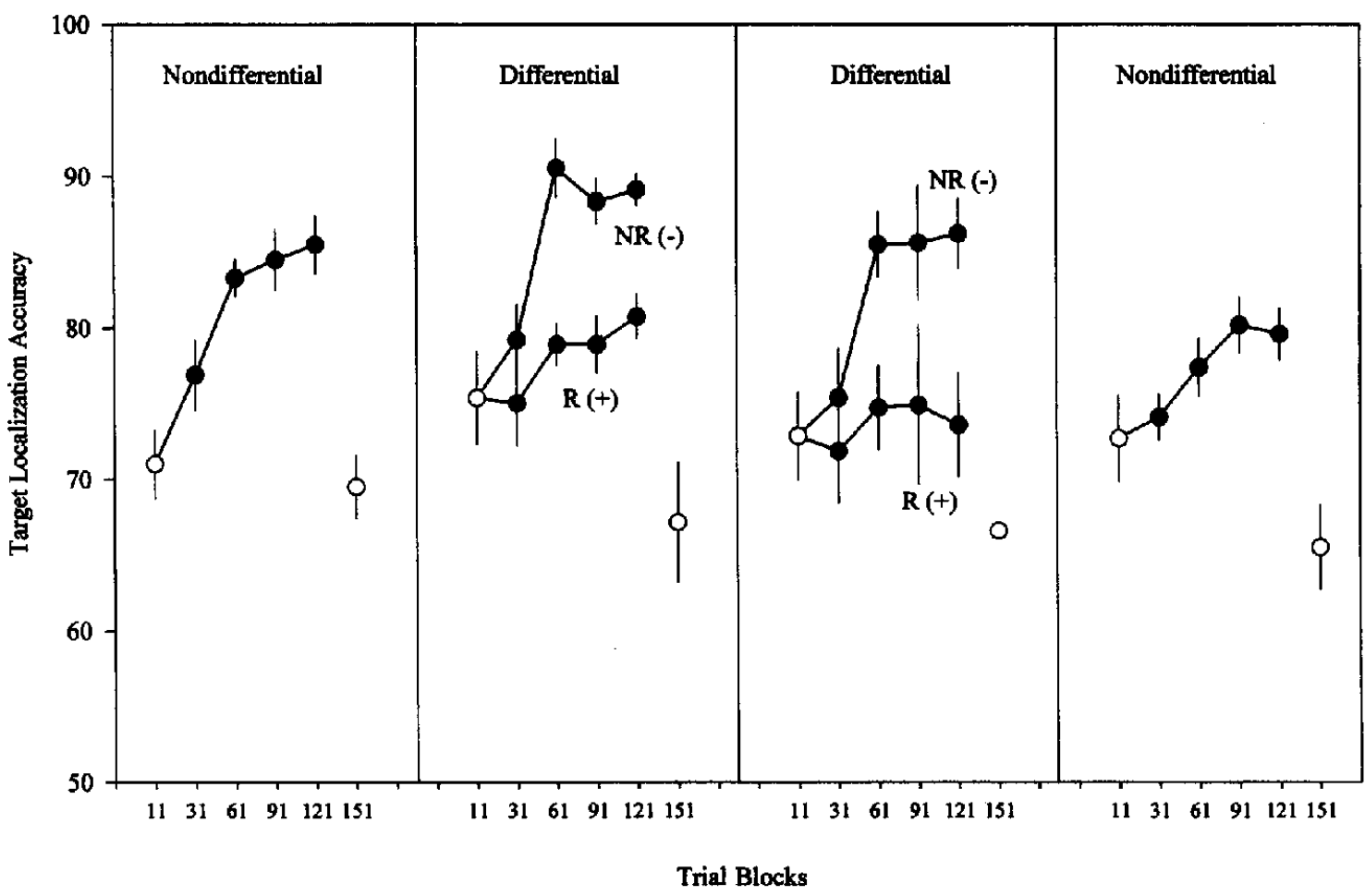

Figure 5. Mean target localization accuracy and standard errors of the mean for the four testing blocks of distractor repetition conducted in Experiment 4. Successive trial blocks are represented along the abscissa for each testing block: 11 (Trials 11-30), 31 (Trials 31-60), 61 (Trials 61-90), 91 (Trials 91-120), 121 (Trials 121-150), 151 (Trials 151-170). During nondifferential testing blocks, the trial blocks for repeated features represent combined performance of 15 trials for each of the two repeated features. Reinforced $\mathbf{R}(+)$ and nonreinforced $\mathrm{NR}(-)$ features are labeled for the differential reinforcement conditions. For baseline (open symbols), only shape performance is represented from their respective testing blocks.

accuracy for the reinforced target feature. Separate two-way repeated-measures ANOVAs-Repeated Feature (reinforced vs. nonreinforced) $\times$ Trial Block (11-30, 31-60, $61-90,91-120,121-150$ ) - were conducted for each differential testing block to examine these interactions. For differential distractor and target repetitions, the Feature $X$ Trial Block interactions were significant in each case, all five $F \mathrm{~s}(4,20)>5.1, p \mathrm{~s}<.005$.

The effects of repetition for the nondifferential blocks were different from those of the differential blocks. For distractor repetition, both features showed a systematic and similar increase in accuracy with nondifferential repetition. Separate one-way repeated-measures ANOVAs (Trial Block: $11-30,31-60,61-90,91-120,121-150$ ) revealed main effects for trial block for nondifferential distractor repetition, both $F \mathrm{~s}(4,20)>3.7, p \mathrm{~s}<.05$. For target repetition, the pattern was more complex. In the first block, there was an increase in accuracy with target repetition, $F(4,20)=3.8$, $p<.05$, although the total increase from baseline [3.9\%; trials (121-150) - trials (11-30)] was less than in the prior differential block $(10.5 \%)$. In the second nondifferential block, however, there was no significant effect of repetition $(F<1)$, although again there was a slight numerical increase from baseline $(2.7 \%)$ across trials. In the third block, the increase across target repetition was stronger $(7.0 \%)$ and significant, $F(4,20)=5.1, p<.005$, and not unlike those seen with the reinforced feature in the differential target conditions (mean increase over all testing blocks from baseline was $8.6 \%$ ) or with nondifferential distractor repetition (mean increase over all testing blocks from baseline was $10.7 \%$ ).

To compare the effects of target repetition across the two reinforcement procedures, we conducted a two-way repeatedmeasures ANOVA - Reinforcement (differential vs. nondifferential) $\times$ Testing Block $(1,2,3)-$ on the difference in accuracy between the last block of rewarded target features (Trials 121-150) and baseline trials (Trials 11-30). For this analysis, the performance on nonreinforced features was not included. This analysis confirmed that the effect of target repetition had a significantly greater increase during differential $(8.6 \%)$ than nondifferential $(4.5 \%)$ target repetition, $F(1$, $5)=14.49, p<.05$. There was no interaction over testing blocks, $F(2,10)=3.14, p<.09$.

To compare the effects of distractor repetition across the two reinforcement procedures, we conducted separate twoway repeated-measures ANOVAs-Reinforcement (Differential vs. Nondifferential) $\times$ Testing Block $(1,2)$ on the difference in accuracy between the last block of rewarded 


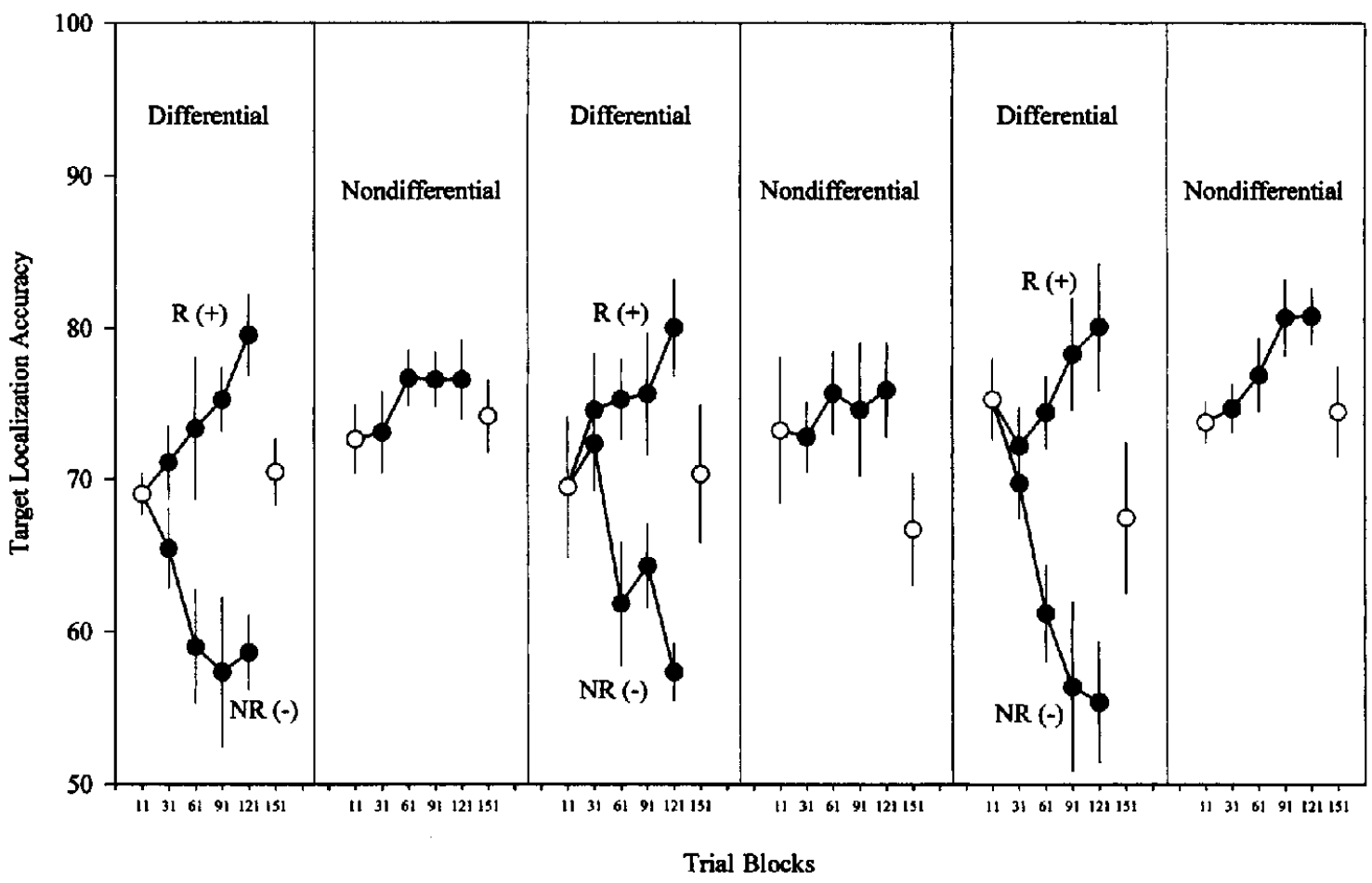

Figure 6. Mean target localization accuracy and standard errors of the mean for the six testing blocks of target repetition conducted in Experiment 4 . Successive trial blocks are represented along the abscissa for each testing block: 11 (Trials 11-30), 31 (Trials 31-60), 61 (Trials 61-90), 91 (Trials 91-120), 121 (Trials 121-150), 151 (Trials 151-170). During nondifferential testing blocks, the trial blocks for repeated features represent combined performance of fifteen trials for each of the two repeated features. Reinforced, $\mathrm{R}(+)$, and nonreinforced, $\mathrm{NR}(-)$, features are labeled for the differential reinforcement conditions. For baseline (open symbols), only shape performance is represented from their respective testing blocks.

distractor features (Trials 121-150) and baseline trials (Trials 11-30) for nondifferential blocks versus reinforced and nonreinforced features during differential testing. These analyses revealed that the effect of distractor repetition was not significantly greater for the nonreinforced feature during differential reinforcement $(13.5 \%)$ in comparison to nondifferential reinforcement $(10.7 \%), F(1,5)=2.0, p>.22$. There was no interaction with testing block, $F(1,5)=1.8$, $p>$.2. The same analysis comparing the effects of nondifferential reinforcement ( $10.7 \%$ increase) to the reinforced feature $(3.1 \%)$ revealed a significant difference, $F(1,5)=$ $6.0, p=.058$.

\section{Discussion}

The most important development during this experiment was the control by repetition exhibited for repeated target features. The best evidence of this was during differential target repetition, which produced a marked increase in accuracy for the repeated and reinforced target feature and a decrease in accuracy for the nonreinforced target feature. That is, the pigeons learned to approach positively reinforced targets and avoid the aversive nonreinforced targets. Given that the identity of the targets was relevant to predicting the potential outcome of each trial, this finding is perhaps not surprising. This effect indicates that the earlier failures in Experiment 1 to see any increase with target repetition were not due to an inability to process target features, but that they were not being processed during those tests. This outcome is consistent with the idea that in the earlier experiments the birds were responding automatically to the target once it was located, but were now attending to the features of this component because of their predictive relations to reinforcement.

Interestingly, a search image-like target repetition effect also emerged during nondifferential target repetition as well. Although the effects were small in the first two testing blocks, by the third block of nondifferential testing the birds were reacting to the two repeated targets in a manner similar to that observed with distractor repetition. The most likely explanation for this effect is a carryover effect of target feature processing from the preceding differential conditions. This suggests that the controlled processing of the target's features was not specifically tied to the presence and absence of reinforcement per se, but it reflects a more generalized activation of target processing by the introduction of these events. It is the increasing recruitment of this activation over repeated differential testing blocks that may explain why the size of the nondifferential target repetition effect grew so by the last testing block.

Besides increasing specific control by target features, 
differential reinforcement had effects on distractor processing, too. First, the nonreinforced feature supported a greater increase in accuracy than did the reinforced distractor feature. Second, and more interesting, the reinforced distractor feature did not generally show the same degree of increased accuracy as a function of distractor repetition as in the comparable nondifferential condition. Why did differential reinforcement modulate distractor repetition in this way? Perhaps part of the answer lies with a need to include an associative component to the analysis. One speculation is that within the temporal context of the potentially aversive nonreinforced feature, there is now the presence of a distractor feature that strongly predicts the possibility of reinforcement (given the target is found). This may cause the reinforced distractor feature to become associatively excitatory. This Pavlovian approach component then competes with the learned operant rule to avoid the distractor region (and preventing a timeout). Thus, the birds occasionally and prematurely peck at the reinforced distractor feature because of its association with target-produced food rather than successfully searching for the odd-item target, with the net result being overall less effective target localization performance.

\section{General Discussion}

The interesting and new finding of the present experiments was that repeating features of the distractor elements resulted in improved target localization in an odd-item search task (Experiments 1, 2, and 4). Experiment 2 found evidence that this distractor repetition effect was mediated by a feature-specific avoidance strategy. Experiment 3 found that the memory underlying this short-term learning effect was long lasting, likely being greater than $30 \mathrm{~min}$. Experiment 4 revealed that differential reinforcement could modulate the presence and form of both target and distractor repetition effects.

These experiments provide clear evidence that featurespecific search strategies are involved in textured visual search. By processing over the short term what not to peck, the pigeons can better avoid repeated distractor items and locate the unknown target more effectively then when these distractor features are highly variable in their identity, such as in the randomized baseline condition. In combination with earlier evidence that relational information is also used in this type of search (Cook, 1992b; see also Cook, Cavoto, \& Cavoto, 1995, 1996), it suggests that multiple processes are simultaneously active in the visual search strategies of pigeons. That is, in the present setting the pigeons' search was governed by both generalized odd-item and a distractorspecific avoidance strategies. It is the flexible combination of these search strategies that allows the present pigeons to successfully discriminate over 48,000 different texture displays. When an odd target region does not readily "pop out" to provoke rapid target responding, the distractor avoidance rule functions to prolong search until a target item or discontinuity is located. It is the increasing feature specificity afforded this distractor avoidance strategy that serves as the foundation for the pigeons' effective use of repetition in the current studies.
The main finding in previous studies of stimulus repetition effects on visual search has been target-mediated effects, where target repetition results in either an accuracy or reaction time benefit (P. M. Blough, 1989, 1991, 1992, 1996; Blough \& Lacourse, 1994; Bond, 1983; Bond \& Riley, 1991; Langley, 1996; Langley et al., 1996; Pietrewicz \& Kamil, 1979; Plaisted, 1997; Reid \& Shettleworth, 1992; Vreven \& Blough, 1998). The major explanations of these findings have clustered around target-focused notions of specific search image or attention to target features. During differential reinforcement of repeated target features, we also found that search could be governed by feature-specific target approach and avoidance strategies, too. By learning specifically which of two target features were paired with reward and which were not, the pigeons were able to approach or avoid these items respectively based on their repetition. When target identity was not specifically tied to different outcomes, however, we did not find the standard search image effect during target repetition. Are these various distractor-specific avoidance and target-specific approach patterns of behavior reflective of complementary changes in the same mechanism? Although very difficult to compare because of the differences in the procedures used in these studies, they are similar in that both represent shortterm changes in the effectiveness of target localization within a single session. The current repetition effects did seem to take longer to develop within a run, but appeared to last longer, then typical of target-mediated search image effects. Further, the distractor avoidance strategy is different from other target-specific approach strategies in that no specific representation of the target's features appeared to be involved in producing its improvement in target localization accuracy.

Interestingly, only one other prior study to our knowledge has found evidence for a distractor-mediated search effect. D. S. Blough (1993) used an odd-item task that contained predictable relations between specific distractor and specific target items. By varying their frequency, he found that pigeons learned conditional expectancies about what targets to look for based on the distractor items present in the displays. Although the mechanisms involved are slightly different (conditional expectancy of the target vs. distractor avoidance), it is interesting that in both the current and in D. S. Blough's (1993) experiments, an odd-item search task was used, and in both cases the pigeons came to rely on the focused processing of the more populous distractor as a critical route to locating the target.

These new results and the previous literature now suggest that there are at least five different tactics used by birds to locate successfully a target in a visual search task. These include the use of generalized relational information and item-specific strategies based on the properties of the target, distractor, and their specific combination. First, birds can use conspicuous oddity information to locate rapidly a unique and clearly visible target (D. S. Blough, 1989; Bond \& Riley, 1991; Cook, 1992b; Reid \& Shettleworth, 1992). We think it is the greater activation of this relational mechanism by the color displays that is responsible for the difference in repetition effects observed for color and shape dimensions in Experiment 1, for instance. Second, birds can use a general- 
ized distractor avoidance rule (Experiments 1, 2, 3, and 4 shape baseline performance). This strategy involves a relational search rule that is based on avoiding distractors (defined by initial encounter, relative size, or number) until a different item or a global target pattern is encountered. This flexible strategy would be highly effective in odd-item tasks in which the target's identity is unknown, and the previously mentioned oddity tactic is prevented by the similarity of the target and distractor items. This generalized distractor avoidance strategy also appears to be the progenitor of yet another way of locating the target. Third, in this case, birds use a feature or item-specific distractor avoidance strategy. It is this tactic that is responsible for the distractor repetition benefits discovered in the present experiments. Fourth, birds can use an item-specific search strategy based on locating a known target item or its features (Experiment 4; P. M. Blough, 1989, 1991, 1996; Bond, 1983; Bond \& Riley, 1991; Langley et al., 1996; Pietrewicz \& Kamil, 1979; Reid \& Shettleworth, 1992). Fifth, birds can use item-specific expectations that have been established between pairs of specific distractors and targets (D. S. Blough, 1993). From this growing list, it might be safe to say that birds potentially use virtually every piece of information available to them in a visual search task in order to locate a relevant target in a background of distractors.

As such, the essential question becomes what conditions cause these different strategies to be used in the various types of visual search tasks that have been studied? Four factors currently seem critical. The first factor is the perceptual discriminability of the target relative to the distractor. The role of perceptual discriminability in search tasks is well established and widely acknowledged. When target items are highly visible, birds are able to directly perceive and easily respond to the target. The specific process underlying these "pop-out" like effects is likely based in the early visual mechanisms for feature registration and perceptual grouping in birds (Cook, 1992a, 1992b, in press; Cook et al., 1996). Only when a target is hard to see, do the other types of search mechanisms outlined above become measurable.

The second factor concerns the number of visual items regularly involved in the task. The role of exemplar number is critical to the successful formation of object categories (Bhatt, Wasserman, Reynolds, \& Knauss, 1988; Kendrick, Wright, \& Cook, 1990) and relational rules (Cook, Katz, \& Cavoto, 1997; Santiago \& Wright, 1984; Wasserman, Hugart, \& Kirkpatrick-Steger, 1995; Wright, Cook, Rivera, Sands, \& Delius, 1988). The same is surely true in the present context as well. As the number of items used to create the set of stimulus displays increases, the more likely control by relational factors will occur. The use of small numbers of stimuli fits more naturally into the pigeons' initial propensity to memorize the specifics of any discrimination. Search conditions using smaller numbers of target or distractor stimuli are thus ideal for generating item-specific search strategies (P. M. Blough, 1989, 1991, 1992, 1996; Blough \& Lacourse, 1994; Bond, 1983; Bond \& Riley, 1991; Langley, 1996; Langley et al., 1996; Pietrewicz \& Kamil, 1979;
Plaisted, 1997; Plaisted \& Mackintosh, 1995; Reid \& Shettleworth, 1992; Vreven \& Blough, 1998).

The third factor appears to be related to the experience of the animals. For instance, we found that the specific properties of the target received very little processing in the current task until the conditions were changed to make the target identity relevant by either reversal discrimination or reward outcomes. When these conditions occurred the birds apparently started to pay attention to target identity. Prior to this, the birds appeared to be responding to the target in an almost "automatic" mode. That is, once it has been localized the birds responded to the target because of the experimental contingencies requiring five pecks, but this pecking behavior had little to do with the birds further processing the target's characteristics. Only when the conditions were changed to make such information more relevant did the birds change their processing to a more controlled mode. Vreven and Blough (1998) have recently reported a related kind of automatic processing effect. They found in a fixed-item visual search task that with extended experience with a specific set of targets that benefits of target repetition diminished under some circumstances. With an increase in the numbers of target stimuli to look for, however, more controlled processing of the displays returned. Given the extensive experience that birds often receive in this type of operant discrimination, perhaps greater experimental attention should be paid to the potential contributions of automatic and controlled processing and responding in such settings.

The fourth factor concerns whether birds are required to solve an odd-item or fixed-item search task. In odd-item searches, visual items are used interchangeably as both targets and distractors. In fixed-item searches, targets and distractors come from different sets of visual items that each have mutually exclusive features. Because of the potential classification of visual items during fixed-item searches, subjects could learn with experience specifically what items they are searching for and which to avoid. If such predictability occurs, then limiting the number of target items should improve search performance because the subject could become certain of the identity of the target to be found. In keeping with this, all previous demonstrations of specific target item repetition effects have involved forms of fixeditem searches (P. M. Blough, 1989, 1991, 1992, 1996; P. M. Blough \& Lacourse, 1994; Bond, 1983; Bond \& Riley, 1991; Langley, 1996; Langley et al., 1996; Pietrewicz \& Kamil, 1979; Plaisted, 1997; Plaisted \& Mackintosh, 1995; Reid \& Shettleworth, 1992; Vreven \& Blough, 1998).

Another factor that may need consideration concerns the possible contributions of conditioning processes to these types of discriminations, especially when item repetition is involved. We found that distractor avoidance, target approach, and target avoidance each were modulated by the nature of the reinforcement contingencies associated with the repetition of these elements. A search image perspective suggests these effects are due to modifications in the amount of attention directed to the repeated elements. A conditioning perspective would suggest these changes could be viewed as a type of short-term learning effect involving 
excitatory approach and inhibitory avoidance responses to the repeated elements. In fact, one way to view the current work is as a series of "mini" acquisition studies within the context of a steady-state discrimination. At the moment, these alternative accounts have not been well separated in studies involving item repetition. It is quite possible that both attention and learning may play a role. One future research direction for this type of research is to isolate the relative contribution of these types of processes to search effects in pigeons.

Finally, the current experiments offer a potentially important and new speculation about the way that foraging animals might search for food. Although much attention has been directed at the formation of specific search images (learning what to look for), far less attention has been focused on learning to ignore or avoid background or substrate characteristics (learning what not to look for). One limitation of forming a search image is that it locks the forager into searching for only a subset of recently encountered items, perhaps at the cost of missing other equally good targets that are present. One benefit of a substrate strategy is that by learning the features of the background that one is currently feeding on, the relative detectability of many different and unknown targets might be simultaneously enhanced for that context. This seems like a potentially adaptive strategy for dealing with the variable nature of edible items that occur within relatively homogeneous patches.

\section{References}

Beck, J. (1966). Effect of orientation and shape similarity on perceptual grouping. Perception \& Psychophysics, 2, 491-495.

Beck, J. (1982). Textural segmentation. In J. Beck (Ed.), Organization and representation in perception (pp. 285-318). Hillsdale, NJ: Erlbaum.

Bhatt, R. S., Wasserman, E. A., Reynolds, W. F., \& Knauss, K. S. (1988). Conceptual behavior in pigeons: Categorization of both familiar and novel examples from four classes of natural and artificial stimuli. Journal of Experimental Psychology: Animal Behavior Processes, 14, 219-234.

Blough, D. S. (1989). Odd-item search in pigeons: Display size and transfer effects. Journal of Experimental Psychology: Animal Behavior Processes, 15, 14-22.

Blough, D. S. (1993). Effects on search speed of probability of target-distractor combinations. Journal of Experimental Psychology: Animal Behavior Processes, 19, 231-243.

Blough, P. M. (1989). Attentional priming and visual search in pigeons. Journal of Experimental Psychology: Animal Behavior Processes, 15, 358-365.

Blough, P. M. (1991). Selective attention and search images in pigeons. Journal of Experimental Psychology: Animal Behavior Processes, 17, 292-298.

Blough, P. M. (1992). Detectability and choice during visual search: Joint effects of sequential priming and discriminability. Animal Learning \& Behavior, 20, 293-300.

Blough, P. M. (1996). Priming during multiple-target search: The cumulative effects of relative target frequency. Animal Learning \& Behavior, 24, 394-400.

Blough, P. M., \& Lacourse, D. M. (1994). Sequential priming in visual search: Contributions of stimulus-driven facilitation and learned expectancies. Animal Learning \& Behavior, 22, 275281.

Bond, A. B. (1983). Visual search and selection of natural stimuli in the pigeon: The attention threshold hypothesis. Journal of Experimental Psychology: Animal Behavior Processes, 9, 292306.

Bond, A. B., \& Riley, D. (1991). Searching image in the pigeon: A test of three hypothetical mechanisms. Ethology, 87, 203-224.

Brown, P. L., \& Jenkins, H. M. (1968). Auto-shaping the pigeon's key peck. Journal of the Experimental Analysis of Behavior, 11, 1-8.

Cook, R. G. (1992a). Dimensional organization and texture discrimination in pigeons. Journal of Experimental Psychology: Animal Behavior Processes, 18, 354-363.

Cook, R. G. (1992b). The acquisition and transfer of texture visual discriminations by pigeons. Journal of Experimental Psychology: Animal Behavior Processes, 18, 341-353.

Cook, R. G. (1992c). The visual perception and processing of textures by pigeons. In W. K. Honig \& G. Fetterman (Eds.), Cognitive aspects of stimulus control (pp. 279-299). Hillsdale, NJ: Erlbaum.

Cook, R. G. (1993a). Gestalt contributions to visual texture discriminations by pigeons. In T. Zentall (Ed.), Animal cognition: A tribute to Donald A. Riley (pp. 251-269). Hillsdale, NJ: Erlbaum.

Cook, R. G. (1993b). The experimental analysis of cognition in animals. Psychological Science, 4, 174-178.

Cook, R. G. (in press). The comparative psychology of avian visual cognition. Current Direction in Psychological Science.

Cook, R. G., Cavoto, K. K., \& Cavoto, B. R. (1995). Same/different texture discrimination and concept learning in pigeons. Journal of Experimental Psychology: Animal Behavior Processes, 21, 253-260.

Cook, R. G., Cavoto, K. K., \& Cavoto, B. R. (1996). Mechanisms of multidimensional grouping, fusion, and search in avian texture discrimination. Animal Learning \& Behavior, 24, 150167.

Cook, R. G., Cavoto, B. R., Katz, J. S., \& Cavoto, K. K. (1997). Pigeon perception and discrimination of rapidly changing texture stimuli. Journal of Experimental Psychology: Animal Behavior Processes, 23, 390-400.

Cook, R. G., Katz, J. S., \& Cavoto, B. R. (1997). Pigeon same-different concept learning with multiple stimulus classes. Journal of Experimental Psychology: Animal Behavior Processes, 23, 417-433.

Cook, R. G., Katz, J. S., \& Cavoto, B. R. (1998). Processes of visual cognition in the pigeon. In S. Soraci \& B. McIlvane (Eds.), Perspectives on fundamental processes in intellectual functioning: Vol. 1. A survey of research approaches. Greenwich, CT: Ablex.

Jenkins, H. M., \& Sainsbury, R. S. (1970). Discrimination learning with the distinctive feature on positive or negative trials. In D. I. Mostofsky (Ed.), Attention: Contemporary theory and analysis (pp. 239-273). New York: Appleton-Century-Crofts.

Julesz, B. (1981). Textons, the elements of texture perception and their interactions. Nature, 290, 91-97.

Kendrick, D. F., Wright, A. A., \& Cook, R. G. (1990). On the role of memory in concept learning by pigeons. Psychological Record, 40, 359-371.

Langley, C. M. (1996). Search images: Selective attention to specific visual features of prey. Journal of Experimental Psychology: Animal Behavior Processes, 22, 152-163.

Langley, C. M., Riley, D. A., Bond, A. B., \& Goel, N. (1996). Visual search for natural grains in pigeons (Columba livia): 
Search images and selective attention. Journal of Experimental Psychology: Animal Behavior Processes, 22, 139-151.

Marr, D. (1982). Vision. San Francisco: W. H. Freeman.

Pietrewicz, A. T., \& Kamil, A. C. (1979, June 22). Search image formation in the blue jay (Cyanocitta cristata). Science, 204, 1332-1333.

Plaisted, K. (1997). The effect of interstimulus interval on the discrimination of cryptic targets. Journal of Experimental Psychology: Animal Behavior Processes, 23, 248-259.

Plaisted, K. C., \& Mackintosh, N. J. (1995). Visual search for cryptic stimuli in pigeons: Implications for the search image and search rate hypotheses. Animal Behaviour, 50, 1219-1232.

QuickBasic 7.0 [Computer software]. (1989). Redmond, WA: Microsoft.

Reid, P. J., \& Shettleworth, S. J. (1992). Detection of cryptic prey: Search image or search rate? Journal of Experimental Psychology: Animal Behavior Processes, 17, 273-286.

Santiago, H. C., \& Wright, A. A. (1984). Pigeon memory: Same/different concept learning, serial probe recognition acquisition, and probe delay effects on the serial-position function. Journal of Experimental Psychology: Animal Behavior Processes, 10, 498-512.
Tinbergen, N. (1960). The natural control of insects in pine woods: I. Factors influencing the intensity of predation by songbirds. Archives Neelandaises de Zoologie, 13, 265-343.

Vreven, D., \& Blough, P. M. (1998). Searching for one or many targets: Effects of extended experience on the runs advantage. Journal of Experimental Psychology: Animal Behavior Processes, 24, 98-105.

Wasserman, E. A., Hugart, J. A., \& Kirkpatrick-Steger, K. (1995). Pigeons show same-different conceptualization after training with complex visual stimuli. Journal of Experimental Psychology: Animal Behavior Processes, 21, 248-252.

Wright, A. A., Cook, R. G., Rivera, J. J., Sands, S. F., \& Delius, J. D. (1988). Concept learning by pigeons: Matching-to-sample with trial-unique video picture stimuli. Animal Learning \& Behavior, 16, 436-444.

Xgraf 5.0 [Computer software]. (1989). Pittsburgh, PA: Komputerwerk.

Received June 2, 1999

Revision received December 8, 1999

Accepted December 8, 1999 